ESAIM: PROCEEDINGS AND SURVEYS, January 2015, Vol. 48, p. 308-320

N. Champagnat, T. Lelièvre, A. Nouy, Editors

\title{
NUMERICAL SIMULATIONS OF THE INVISCID BURGERS EQUATION WITH PERIODIC BOUNDARY CONDITIONS AND STOCHASTIC FORCING
}

\author{
Emmanuel Audusse $^{1}$, SÉbastien Boyaval ${ }^{2}$, Yueyuan GaO $^{3}$ and Danielle Hilhorst ${ }^{4}$
}

\begin{abstract}
We perform numerical simulations in the one-dimensional torus for the first order Burgers equation forced by a stochastic source term with zero spatial integral. We suppose that this source term is a white noise in time, and consider various regularities in space. For the numerical tests, we apply a finite volume scheme combining the Godunov numerical flux with the Euler-Maruyama integrator in time. Our Monte-Carlo simulations are analyzed in bounded time intervals as well as in the large time limit, for various regularities in space. The empirical mean always converges to the space-average of the (deterministic) initial condition as $t \rightarrow \infty$, just as the solution of the deterministic problem without source term, even if the stochastic source term is very rough. The empirical variance also stablizes for large time, towards a limit which depends on the space regularity and on the intensity of the noise.
\end{abstract}

Résumé. Nous effectuons une étude numérique de l'équation de Burgers non visqueuse en dimension un d'espace, avec des conditions aux limites périodiques et un terme source stochastique de moyenne spatiale nulle. Ce terme source possède la régularité d'un bruit blanc en temps, tandis que nous considérons différentes régularités en espace. Pour les tests numériques, nous utilisons un schéma de volumes finis combinant une intégration en temps de type Euler-Maruyama avec le flux numérique de Godunov. Nous effectuons des simulations avec la méthode de Monte-Carlo et analysons les résultats pour différentes régularités en accordant une attention particulière au comportement en temps long. Il apparaît que la moyenne empirique des réalisations converge toujours vers la moyenne en espace de la condition initiale (déterministe) quand $t \rightarrow \infty$, comme c'est le cas pour la solution du problème sans terme source, même dans le cas où le terme stochastique est peu régulier en espace. Par ailleurs, la variance empirique converge elle aussi en temps long, vers une valeur qui dépend de la régularité et de l'amplitude du terme stochastique.

\section{InTRODUCTION}

Our aim is to numerically approximate solutions of the stochastically forced inviscid Burgers equation

$$
\frac{\partial u}{\partial t}+\frac{\partial}{\partial x}\left(\frac{u^{2}}{2}\right)=g
$$

in a bounded domain of unit length with periodic boundary conditions or, equivalently, on the torus $x \in \mathbb{S}^{1}$.

We suppose that the stochastic source term in (1) has zero space average

$$
\int_{\mathbb{S}^{1}} g=0
$$

in order to preserve the conservative character of the inviscid Burgers equation on the torus. Indeed, given a probability space $(\Omega, \mathcal{F}, \mathbb{P})$, we consider the Cauchy problem for (1) together with a deterministic initial condition

\footnotetext{
${ }^{1}$ Université Paris 13, Institut Galilée, 99 avenue Jean-Baptiste Clément, 93430 Villetaneuse, France, e-mail: eaudusse@yahoo.fr

${ }^{2}$ Université Paris Est, Laboratoire d'hydraulique Saint-Venant (Ecole des Ponts ParisTech - EDF R\& D - CEREMA), 6 quai Watier, 78401 Chatou, France \& INRIA Rocquencourt, MATHERIALS, e-mail: sebastien.boyaval@enpc.fr

${ }^{3}$ Laboratoire de Mathématiques, CNRS et Université de Paris-Sud, 91405 Orsay Cedex, France, e-mail: yueyuan.gao@math.u-psud.fr

${ }^{4}$ Laboratoire de Mathématiques, CNRS et Université de Paris-Sud, 91405 Orsay Cedex, France, e-mail: danielle.hilhorst@math.upsud.fr
}

(C) EDP Sciences, SMAI 2015 
$u(\cdot, t=0)=u_{0}$. Then, (2) implies that solutions satisfy the conservation property

$$
\int_{\mathbb{S}^{1}} u=\int_{\mathbb{S}^{1}} u_{0} \quad \forall t>0 .
$$

Moreover, the stochastic source term $g$ is assumed to behave as a white noise with respect to time $t \in[0, T)$. Then, the exact interpretation of (1) is not obvious. We recall that in the deterministic case where $g$ is a given smooth function of time and space (continuously differentiable for instance, see e.g. [17]), only discontinuous solutions to the Cauchy problem for (1) can be rigorously defined for large time (discontinuities appear even if the initial condition is smooth), and a notion of entropic solution is necessary for uniqueness. In the case that $g$ is stochastic, further notions of solutions are necessary $[9,10]$.

In this note, we consider space-time discrete versions of (1). Considering the inherent difficulties of (1), our numerical scheme combines standard discretization techniques for scalar first order conservation laws such as the inviscid Burgers equation with periodic boundary conditions $[5,18]$ together with standard discretization techniques for stochastic differential equations.

More precisely, we consider a finite volume discretization. Given $I \in \mathbb{N}^{\star}$, we split the one-dimensional torus $\mathbb{S}^{1}$ into cells of uniform volume $\Delta x:=1 / I$. Then, a discretization of (1) between two times $0<t^{n}<t^{n+1}$ is typically obtained from the integral formula

$$
\begin{aligned}
\int_{(i-1 / 2) \Delta x}^{(i+1 / 2) \Delta x} u\left(x, t^{n+1}\right) d x & =\int_{(i-1 / 2) \Delta x}^{(i+1 / 2) \Delta x} u\left(x, t^{n}\right) d x \\
& +\int_{t^{n}}^{t^{n+1}}\left(\left(u^{2} / 2\right)((i-1 / 2) \Delta x, t)-\left(u^{2} / 2\right)((i+1 / 2) \Delta x, t)\right) d t+\int_{t^{n}}^{t^{n+1}} \int_{(i-1 / 2) \Delta x}^{(i+1 / 2) \Delta x} g d x d t
\end{aligned}
$$

by defining a numerical solution $u^{n}$ with a fixed time step $\Delta t=\Delta t^{n}:=t^{n+1}-t^{n}>0$ for all $n \in\left\{0,1, \ldots, N_{T}-1\right\}$ with $N_{T}=T / \Delta t$ through the relation

$$
\frac{u_{i}^{n+1}-u_{i}^{n}}{\Delta t}=\frac{1}{\Delta x}\left(F_{i+1 / 2}^{n}-F_{i-1 / 2}^{n}\right)+\alpha \sqrt{\frac{1}{\Delta x \Delta t}} G_{i}^{n} \quad \text { for all } n \in\left\{0,1, \ldots, N_{T}-1\right\} \text { and } i \in\{1, \ldots, I\},
$$

where the positive constant $\alpha$ represents the intensity of the noise and the space derivative is approximated in a conservative way through the definition of the fluxes $F_{i+1 / 2}^{n}[18]$; the time process is discretized by an explicit Euler-Maruyama time integrator $[16,20]$, recalling that $g$ is a white noise in time. We have thereby defined a Markov process $\left(u^{n}\right)_{n \in \mathbb{N}}$ with values in $\mathbb{R}^{I}$ to discretize the cell-averages of the solution of (1), starting with the finite volume approximation of the deterministic initial condition $u^{0}$

$$
u_{i}^{0}=\frac{1}{\Delta x} \int_{(i-1 / 2) \Delta x}^{(i+1 / 2) \Delta x} u_{0}(x) .
$$

We make precise definitions of the flux $F_{i+1 / 2}^{n}$ and of the stochastic source term $G_{i}^{n}$ in the next section.

A first important question is whether, and how, the Markov process defined by (4) allows to approximate solutions of (1). In the deterministic case with $G^{n}=0$, our discrete sequences $\left(u^{n}\right)_{n \in \mathbb{N}}$ are already known to converge for all $T>0$, as the space and time steps tend to zero under a CFL condition [13,21], toward the unique entropic solution of the inviscid Burgers equation with periodic boundary conditions

$$
\frac{\partial u}{\partial t}(x, t)+\frac{\partial}{\partial x}\left(\frac{u^{2}}{2}(x, t)\right)=0 \quad \text { for all }(x, t) \in \mathbb{S}^{1} \times[0, T) .
$$

But in order to be able to consider stochastic cases where $G^{n} \neq 0$, one still needs to define a notion of solution to (1). For the "space-time white noise" case in the sense of zero correlation-length in space and time, which is among the cases numerically approximated in this work, we are not aware of any existing mathematical notion of solution. A univoque stochastic entropic solution has however already been rigorously defined in the case of a colored noise where $g$ has sufficiently regular space variation.

In our one-dimensional setting with additive stochastic forcing, solutions of the viscous stochastically forced Burgers equation, that is with an additional diffusion term $-\nu \partial_{x x}^{2} u$ on the left-hand-side of $(1)(\nu>0)$, have been shown to trajectorially (i.e. for each realization) converge to the entropic solutions when $\nu \rightarrow 0$, as in the 
deterministic case. Note that the viscous stochastically forced Burgers equation has been much more studied than the inviscid one, from the pure theoretical as well as numerical [1] viewpoints. Additionally, let us mention [14] where stochastic entropic solutions to one-dimensional scalar conservation laws have been univoquely defined. In [9], kinetic solutions have been recently defined in any dimension (they coincide with the former in the case of space dimension one). Moreover we refer to [10] for a Hamilton-Jacobi reformulation of Burgers equation.

However, it is not clear whether, and how, a conservative numerical scheme as ours does indeed approximate solutions. In particular, it has been shown in [15] that the discretization of the nonlinear term has a lot of impact on the continuous limit of the viscous stochastic case. Although we use here a more usual discretization of the nonlinear term (flux) than in [15], which actually allows one to define the entropic solutions of Burgers equation in the deterministic case, we are not able to pass to the limit $\Delta t \rightarrow 0$ yet. During the completion of the present work, we remarked that approximations by a flux-splitting scheme (i.e. a special choice of the numerical flux) have been recently shown to converge to the stochastic entropic solution of a scalar conservation law under a fixed CFL condition on the time step [3], in the case of a multiplicative noise which vanishes when $u=0$.

Another important question with respect to the stochastic dynamics is the behaviour of $u^{n}$ as $n \rightarrow \infty$, with the discretization of (1) being fixed. Note that, in deterministic cases without noise [7] or with a time-independent forcing with zero space average [2], the solutions of (5) are indeed already known to converge for large times to the constant $\int_{\mathbb{S}^{1}} u_{0}(x) d x$. Moreover, the existence of an invariant measure has been proved for sufficiently regular noise in space, using the equivalence with a Hamilton-Jacobi equation in [10], and with kinetic formalism in [8]. In this work, we numerically study the large time behaviour when the noise $G^{n}$ is defined with various regularities in space and various intensities.

The stochastic Burgers equation with periodic boundary conditions has already been studied numerically by numerous authors, including its long-time statistics (usually from a physical more than mathematical point of view). Let us mention [11,12], where some interesting physical motivations are explained. The numerical scheme which we propose here does not seem to have been used yet, in particular for large time simulations.

\section{NUMERICAL METHOD}

We define the two-point numerical flux $\left(u^{2} / 2\right)((i-1 / 2) \Delta x, t)$ by the Godunov scheme [18]

$$
F_{i-1 / 2}=\left\{\begin{array}{lll}
\frac{u_{i}^{2}}{2} & \text { if } & u_{i-1} \leq u_{i} \leq 0 \\
\frac{u_{i-1}^{2}}{2} & \text { if } & 0 \leq u_{i-1} \leq u_{i} \\
0 & \text { if } & u_{i-1} \leq 0 \leq u_{i} \\
\frac{u_{i-1}^{2}}{2} & \text { if } & u_{i} \leq u_{i-1} \text { and } \frac{u_{i}+u_{i-1}}{2} \geq 0 \\
\frac{u_{i}^{2}}{2} & \text { if } & u_{i} \leq u_{i-1} \text { and } \frac{u_{i}+u_{i-1}}{2} \leq 0 .
\end{array}\right.
$$

This flux is known to be consistent in a weak sense, see e.g. $[5,13]$. Furthermore, in the deterministic case with a smooth source term $G_{i}^{n}$, the numerical scheme is stable (i.e. entropy-satisfying) under the classical CFL condition

$$
\Delta t^{n} \leq \frac{\Delta x}{\max _{i \in\{1,2, . . I\}}\left\{\left|u_{i}^{n}\right|\right\}},
$$

so that the numerical solution converges to the unique entropy solution of $(1)$ on $[0, T)$ for all $T>0$ as $\max _{n=0 \ldots N_{T}-1} \Delta t^{n} \rightarrow 0$, see e.g. [6].

We now turn to the stochastic source term $g$. On the one hand, we would like the function $t \rightarrow g(x, t)$ to have the regularity of a white noise for all $x$, because this is a natural source term from the physical viewpoint. On the other hand, a notion of stochastic entropic solution has only been defined when the function $x \rightarrow g(x, t)$ is smooth enough. In this note, we would like to explore numerically our model with various source terms of different space regularities. Restricting to odd integers $I$, we define the discrete noise as the finite volume approximation

$$
G_{i}^{n}=\sqrt{\frac{2}{I}}\left(\sum_{k=1}^{\frac{I-1}{2}}\left(\frac{C_{k}^{n}}{k^{\beta}} \cos (2 \pi k \Delta x i)-\frac{S_{k}^{n}}{k^{\beta}} \sin (2 \pi k \Delta x i)\right)\right)
$$


of a Fourier series where $C_{k}^{n}$ and $S_{k}^{n}$, in the Fourier coefficients $C_{k}^{n} / k^{\beta}, S_{k}^{n} / k^{\beta}$, are mutually independent identically distributed (i.i.d.) random variables with normal distribution $\mathcal{N}(0,1)$ for all $k$ and $n$, where $\mathcal{N}\left(\mu, \sigma^{2}\right)$ denotes the normal distribution with expected value $\mu$ and variance $\sigma^{2}$, and where the parameter $\beta$ controls the regularity of the variations with respect to space: $\beta=0$ corresponds to white noise in space whereas $\beta>0$ corresponds to colored noise. The well-posedness theory for the corresponding continuous problem is only valid for $\beta>\frac{1}{2}$ [8]. We will check in the appendix that if $\beta=0$,

$$
\mathbb{E}\left(G_{i}^{n} G_{j}^{m}\right)=\delta_{m, n}\left[\delta_{i, j}-\frac{1}{I}\right]
$$

We will also check that for all $\beta \geq 0, \sum_{i=1}^{I} G_{i}^{n}=0$ for all $n$, which is the discrete counterpart of the conservativity requirement $\int_{\mathbb{S}^{1}} g=0$ on the source term. Note that the discrete noise (8) has the same scaling in $\Delta t^{n}$ and $\Delta x$ as in $[14,15]$.

We compute the empirical mean and variance estimators of the Markov chain entries (4) by the Monte-Carlo method

$$
\mathrm{E}_{M}\left(u_{i}^{n}\right):=\frac{1}{M} \sum_{m=1}^{M} u_{i}^{n}\left(\omega_{m}\right), \quad \operatorname{Var}_{M}\left(u_{i}^{n}\right):=\frac{1}{M} \sum_{m=1}^{M}\left|u_{i}^{n}\left(\omega_{m}\right)-\mathrm{E}_{M}\left(u_{i}^{n}\right)\right|^{2},
$$

invoking $M$ i.i.d. realizations $u_{i}^{n}\left(\omega_{m}\right), m=1 \ldots M$, computed with $I N M$ Gaussian numbers $\mathcal{N}(0,1)($ cf. $(8))$. Here $N$ denotes the number of time iterations that one needs for the average of the realizations to reach the stationary solution zero of the deterministic problem with a good precision, see next section.

The number of volume elements is first fixed equal to $I=101$, so that the number of Fourier modes in (8) is equal to $I-1=100$ (recall that the constant mode is eliminated in order to ensure a zero space average).

The CFL condition (7) is naturally stochastic, i.e. dependent on the realization. To maintain a fixed number of time steps for each realization, we assume that $\left|u_{i}^{n}\right| \leq \bar{u}$ with $\bar{u}:=10$ set arbitrarily, which allows to fix the CFL condition as $\Delta t=0.1 \Delta x$. Of course, we are aware that this may introduce a bias in the probability law which is simulated.

We have performed numerical tests with a variety of values of $\alpha$ and $\beta$. In each case, we chose the number of realizations $M$ large enough so that confidence intervals for $\mathrm{E}_{M}\left(u_{i}^{n}\right)$ and $\operatorname{Var}_{M}\left(u_{i}^{n}\right)$ are small enough, and our assumption $\left|u_{i}^{n}\right| \leq \bar{u}$ was hardly violated (at a frequency less than $1 / 2000$ ). However, we reject the realization in the case that $\max _{i \in\{1,2, \ldots I\}}\left\{\left|u_{i}^{n}\right|\right\}>10$ for some $n \in\{1, \ldots, N\}$.

The influence of the number of realizations on the evaluations of $\left\|\operatorname{Var}_{U}(x)\right\|_{L^{1}(0,1)}(t)$, in the cases that $\alpha=1$, $\beta=0$ and $\alpha=1, \beta=1$ with initial condition $u_{0}(x)=\sin (2 \pi x)$ is presented in Figure 1.

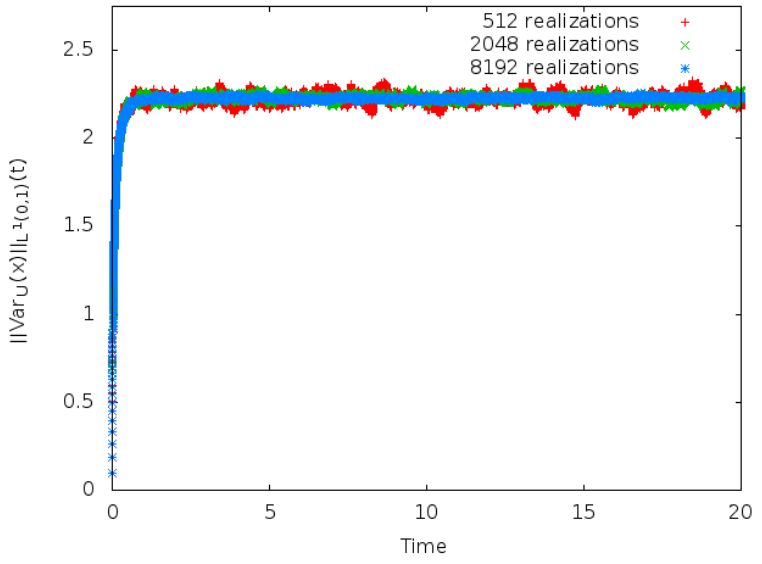

(A) $\alpha=1$ and $\beta=0$

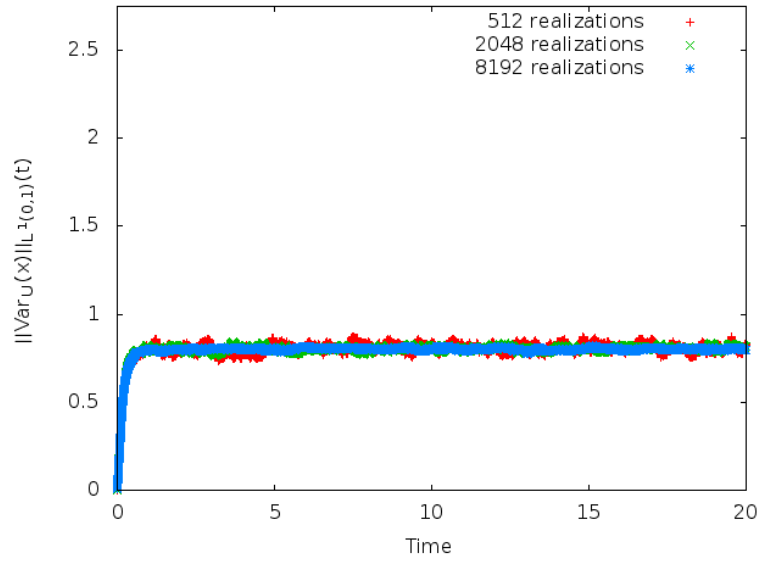

(B) $\alpha=1$ and $\beta=1$

Figure 1. Time evolution of the $L^{1}$ norm of the variance

It is remarkable that large time convergence seems to occur even when $\beta<\frac{1}{2}$. However, to conclude about the continuous limit, one should still discuss the space and time discretization in those cases. Moreover, a complete understanding of ergodic properties would require the study of higher-order moments as well. 
In order to discuss the number of realizations, we define the random variable $X_{m}$ for the $m$-th realization by

$$
X_{m}=\int_{0}^{1}\left|u\left(\omega_{m}\right)(x, T)\right| d x .
$$

For each realization, we use the same scheme. Thus if we perform $M$ realizations, the series $\left\{X_{m}\right\}_{\{m=1,2, \ldots M\}}$ is independently and identically distributed. We denote the corresponding expectation by $\bar{\mu}$ and its variance by $\bar{\sigma}^{2}$.

According to the central limit theorem, there holds

$$
\mathbb{P}\left(-z \leq \frac{\frac{1}{M} \sum_{m=1}^{M} X_{m}-\bar{\mu}}{\bar{\sigma}^{2} / M} \leq z\right) \underset{M \rightarrow+\infty}{\longrightarrow} \int_{-z}^{z} \frac{e^{-t^{2} / 2}}{\sqrt{2 \pi}} d t \quad \forall z \in \mathbb{R}
$$

The confidence interval $\left[I_{1}(M), I_{2}(M)\right]$ of $\bar{\mu}$ at the confidence level $1-p$ is given by

$$
\left[I_{1}(M), I_{2}(M)\right]=\left[\frac{1}{M} \sum_{m=1}^{M} X_{m}-z_{p} \cdot \sqrt{\bar{\sigma}^{2} / M}, \frac{1}{M} \sum_{m=1}^{M} X_{m}+z_{p} \cdot \sqrt{\bar{\sigma}^{2} / M}\right]
$$

where the quantile $z_{p}$ is such that $\int_{-z_{p}}^{z_{p}} \frac{e^{-t^{2} / 2}}{\sqrt{2 \pi}} d t=1-p$. Since the variance $\bar{\sigma}^{2}$ is unknown, we need to approximate it by:

$$
\bar{\sigma}^{2} \approx \operatorname{Var}^{M}(X):=\frac{1}{M} \sum_{m=1}^{M} X_{m}^{2}-\left(\frac{1}{M} \sum_{m=1}^{M} X_{m}\right)^{2}
$$

for a large enough value of $M$. Thus

$$
\left[I_{1}(M), I_{2}(M)\right] \approx\left[\frac{1}{M} \sum_{m=1}^{M} X_{m}-z_{p} \cdot \sqrt{\frac{\operatorname{Var}^{M}(X)}{M}}, \frac{1}{M} \sum_{m=1}^{M} X_{m}+z_{p} \cdot \sqrt{\frac{\operatorname{Var}^{M}(X)}{M}}\right]
$$

We fix $\alpha=1, \beta=0, T=20$. We choose $95 \%$ as the confidence level, which corresponds to $z_{0.05} \approx 1.96$.
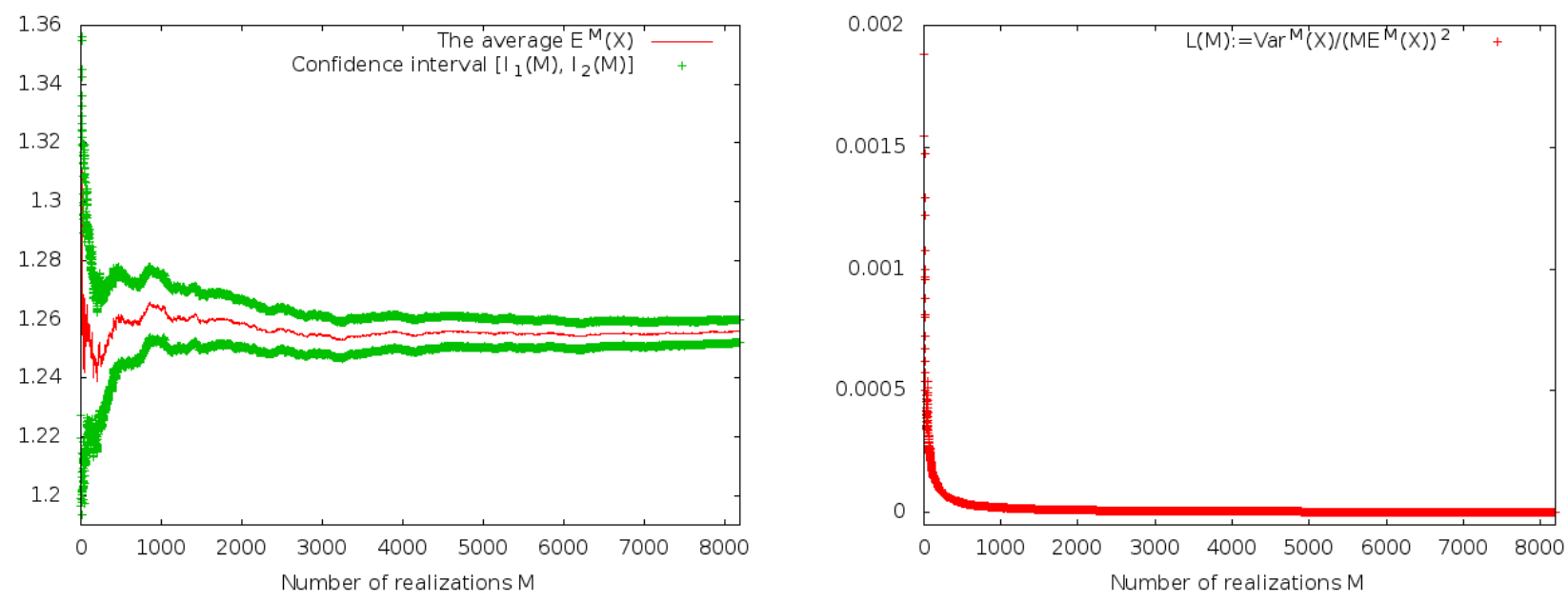

Figure 2. Confidence interval of $\bar{\mu}$ at level 0.95

On the left-hand side, the average $\mathrm{E}^{M}(X):=\frac{1}{M} \sum_{m=1}^{M} X_{m}$ and the confidence interval $\left[I_{1}(M), I_{2}(M)\right]$ are plotted as functions of $M$, while the function $L(M):=\frac{\operatorname{Var}^{M}(X)}{M\left(\mathrm{E}^{M}(X)\right)^{2}}$ is plotted on the right-hand figure. It turns out that $\frac{\operatorname{Var}^{M}(X)}{M \cdot\left(\mathrm{E}^{M}(X)\right)^{2}}$ is a decreasing function of $M$ and that $\frac{\operatorname{Var}^{8192}(X)}{8192\left(\mathrm{E}^{8192}(X)\right)^{2}} \approx 2.5 \times 10^{-6}$.

Next, we study the second moment of $\left\{X_{m}\right\}_{m=1,2, \ldots M}$. We recall that $\left\{X_{m}\right\}_{m=1,2, \ldots M}$ is independently and identically distributed, so that we can use the notations $\mathrm{E}\left(X_{m}^{2}\right)$ for the second moment and $\operatorname{Var}\left(X_{m}^{2}\right)$ for the 
corresponding variance for all $m=1,2, \ldots, M$. According to (11) and (12), the confidence interval of $\mathrm{E}\left(X_{m}^{2}\right)$, namely $\left[I_{3}(M), I_{4}(M)\right]$, is given by

$$
\left[I_{3}(M), I_{4}(M)\right]=\left[\frac{1}{M} \sum_{m=1}^{M} X_{m}^{2}-z_{p} \cdot \sqrt{\operatorname{Var}\left(X_{m}^{2}\right) / M}, \frac{1}{M} \sum_{m=1}^{M} X_{m}^{2}+z_{p} \cdot \sqrt{\operatorname{Var}\left(X_{m}^{2}\right) / M}\right]
$$

where $\operatorname{Var}\left(X_{m}^{2}\right)=\mathrm{E}\left(\left(X_{m}^{2}\right)^{2}\right)-\left(\mathrm{E}\left(X_{m}^{2}\right)\right)^{2}$, which we approximate by

$$
\operatorname{Var}\left(X_{m}^{2}\right) \approx \operatorname{Var}^{M}\left(X^{2}\right):=\frac{1}{M} \sum_{m=1}^{M} X_{m}^{4}-\left(\frac{1}{M} \sum_{m=1}^{M} X_{m}^{2}\right)^{2}
$$

We fix $\alpha=1, \beta=0, T=20$. We choose $95 \%$ as the confidence level, which corresponds to $z_{0.05} \approx 1.96$.
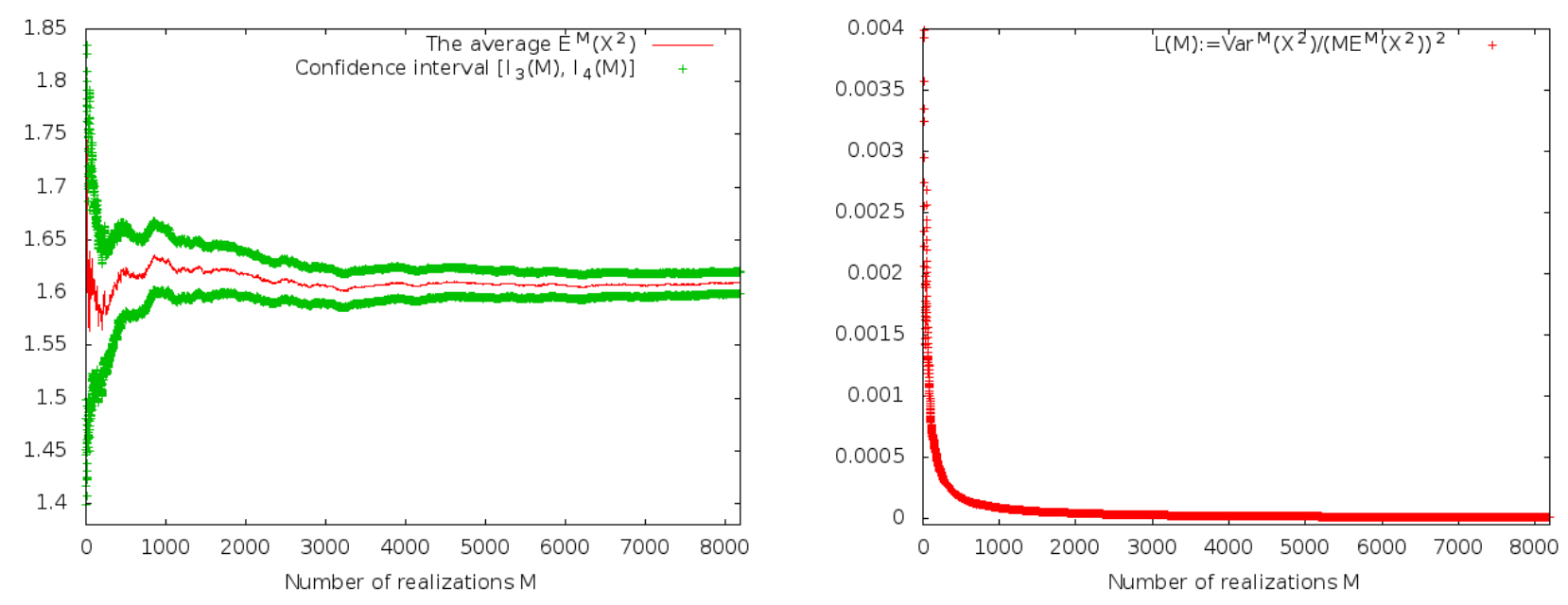

Figure 3. Confidence interval of $\mathrm{E}\left(X_{m}^{2}\right)$ at level 0.95

On the left-hand side, the average $\mathrm{E}^{M}\left(X^{2}\right):=\frac{1}{M} \sum_{m=1}^{M} X_{m}^{2}$ and the confidence interval $\left[I_{3}(M), I_{4}(M)\right]$ are plotted as functions of $M$, while the function $L(M):=\frac{\operatorname{Var}^{M}\left(X^{2}\right)}{M\left(\mathrm{E}^{M}\left(X^{2}\right)\right)^{2}}$ is plotted on the right-hand figure. It turns out that $\frac{\operatorname{Var}^{M}\left(X^{2}\right)}{M \cdot\left(\mathrm{E}^{M}\left(X^{2}\right)\right)^{2}}$ is a decreasing function of $M$ and that $\frac{\operatorname{Var}^{8192}\left(X^{2}\right)}{8192\left(\mathrm{E}^{8192}\left(X^{2}\right)\right)^{2}} \approx 1.04 \times 10^{-5}$.

\section{Results AND Discussion}

The numerical tests are all performed with the deterministic initial condition $u_{0}(x)=\sin (2 \pi x), x \in \mathbb{S}^{1}$. We performed 8192 realizations for each computation. In the figures 4,5 and 6 , we present on the left-hand side comparisons between $\mathrm{E}_{M}\left(u_{i}^{n}\right)$, and $\bar{u}_{i}^{n}$ in the case without noise, for different values of the discrete time $t^{n}=n \Delta t$. On the right-hand side, we show comparisons between $u_{i}^{n}$ for some realization and $\bar{u}_{i}^{n}$ in the case without noise, at the same values of the discrete time $t^{n}=n \Delta t$.

We first fix three cases $\alpha=0.1, \beta=0, \alpha=0.1, \beta=1$ and $\alpha=0.1, \beta=2$. 

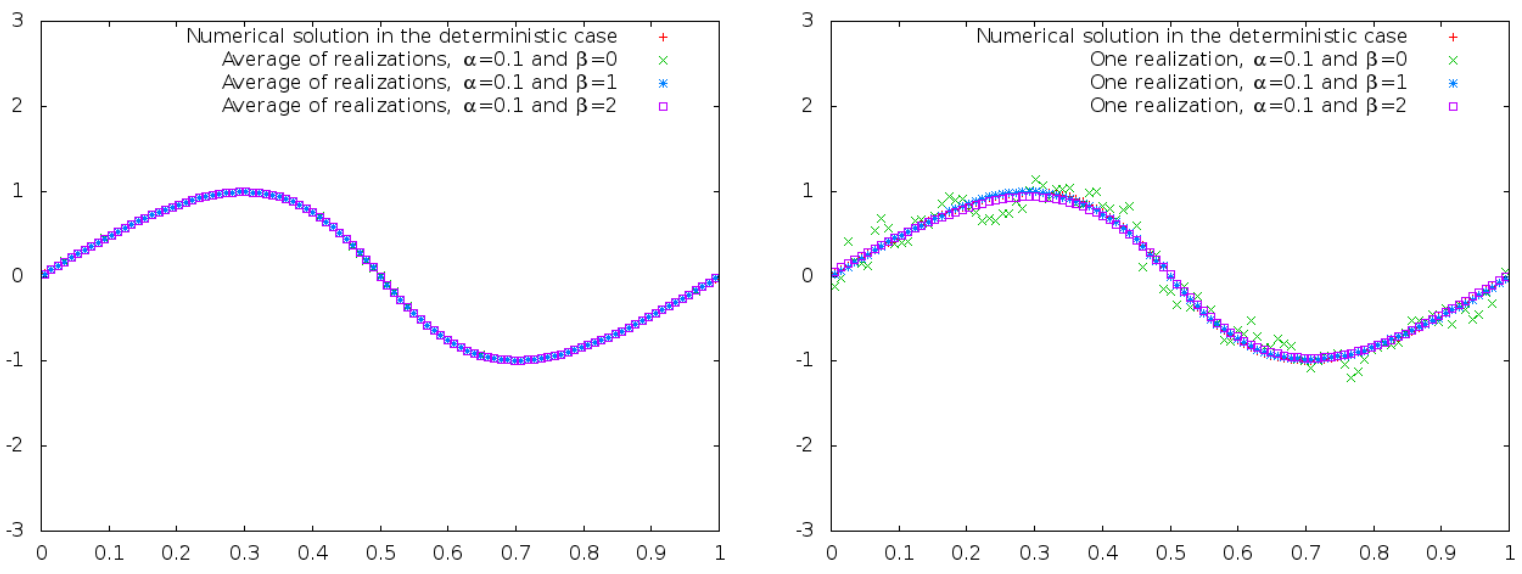

Figure 4. Empirical average (left) and one realization (right) at $t=0.05$
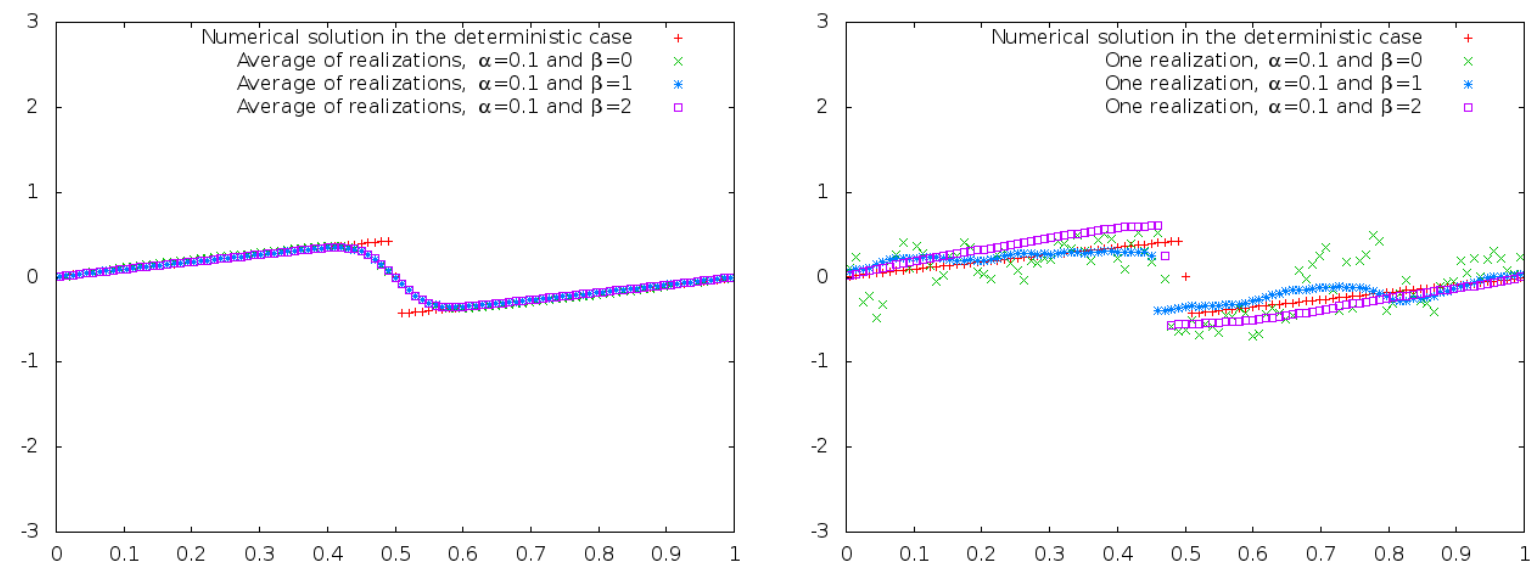

Figure 5. Empirical average (left) and one realization (right) at $t=1$
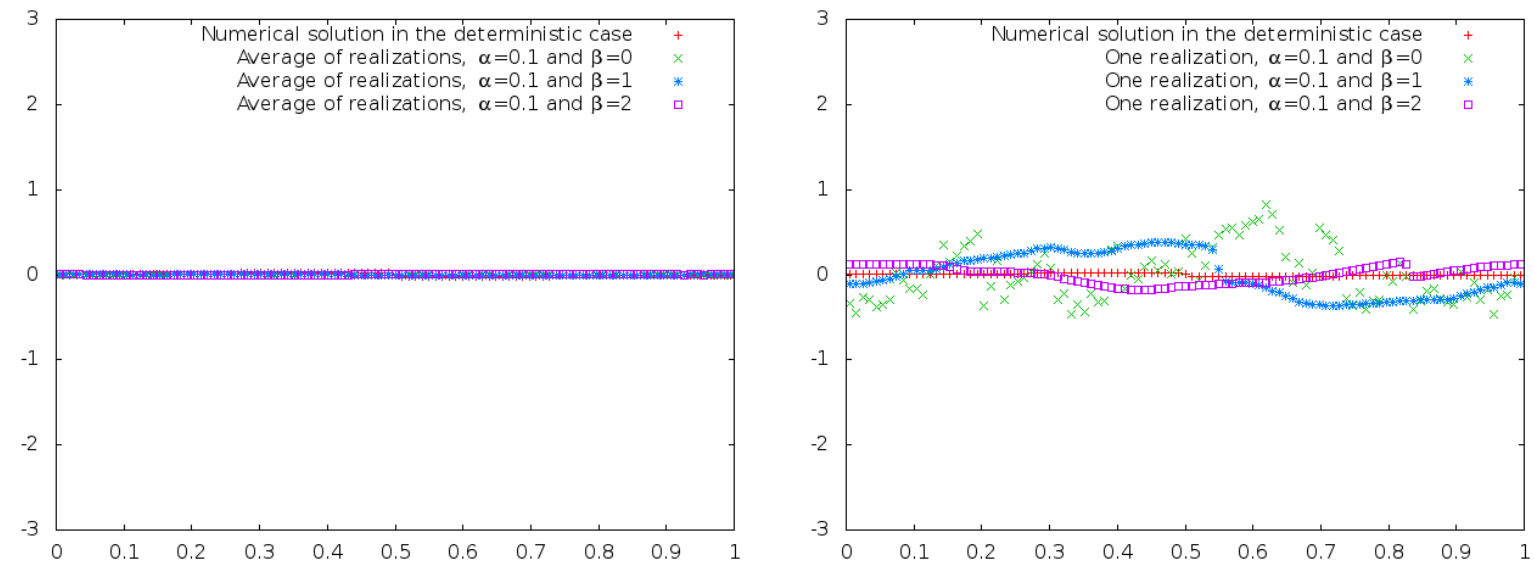

FIGURE 6. Empirical average (left) and one realization (right) at $t=20$

In Figure 7 we rescale the comparisons for the empirical average at the times $t=1$ and $t=20$. 


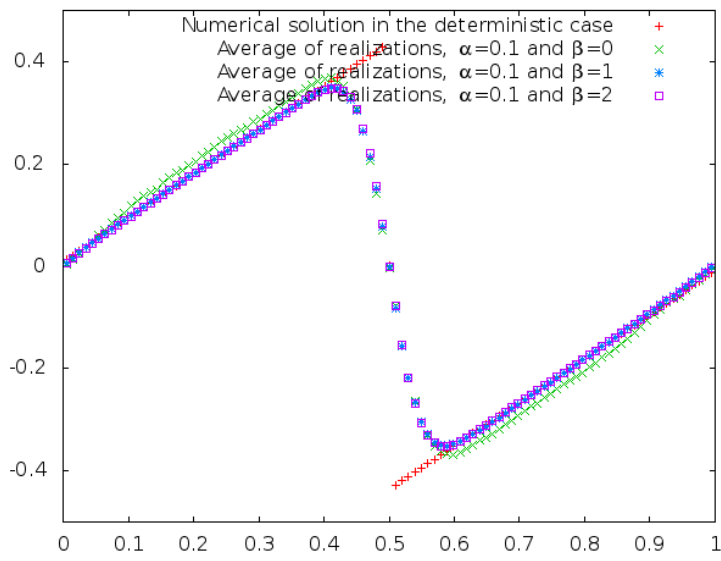

(A) $t=1$

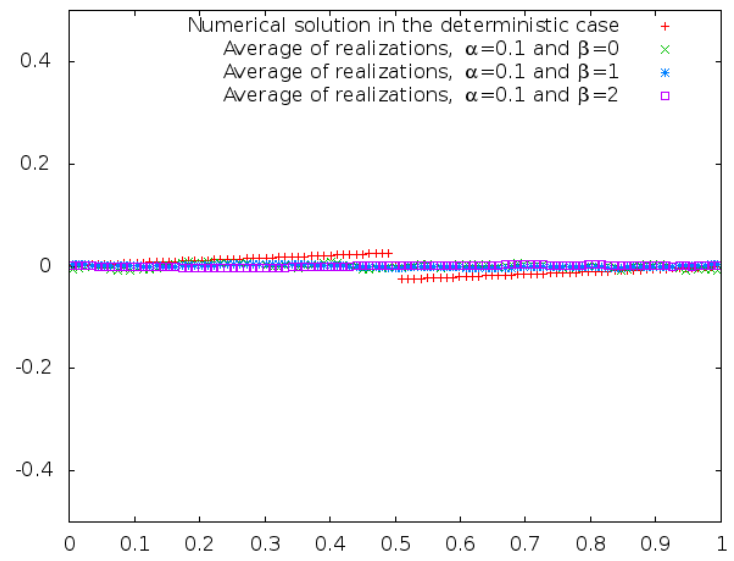

(в) $t=20$

Figure 7. Rescaled graphs for $t=1$ and $t=20$

We then fix $\alpha=1, \beta=0, \alpha=1, \beta=1$ and $\alpha=1, \beta=2$. We present the corresponding results in the figures 8 , 9 and 10.
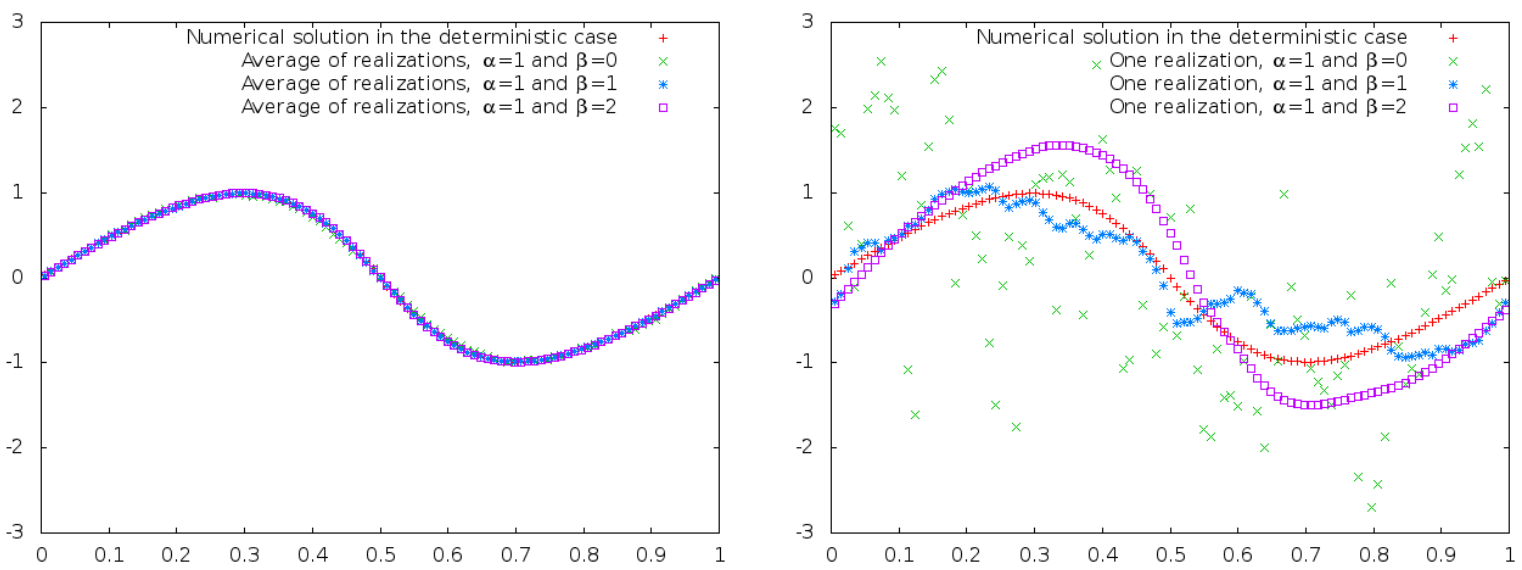

Figure 8. Empirical average (left) and one realization (right) at $t=0.05$
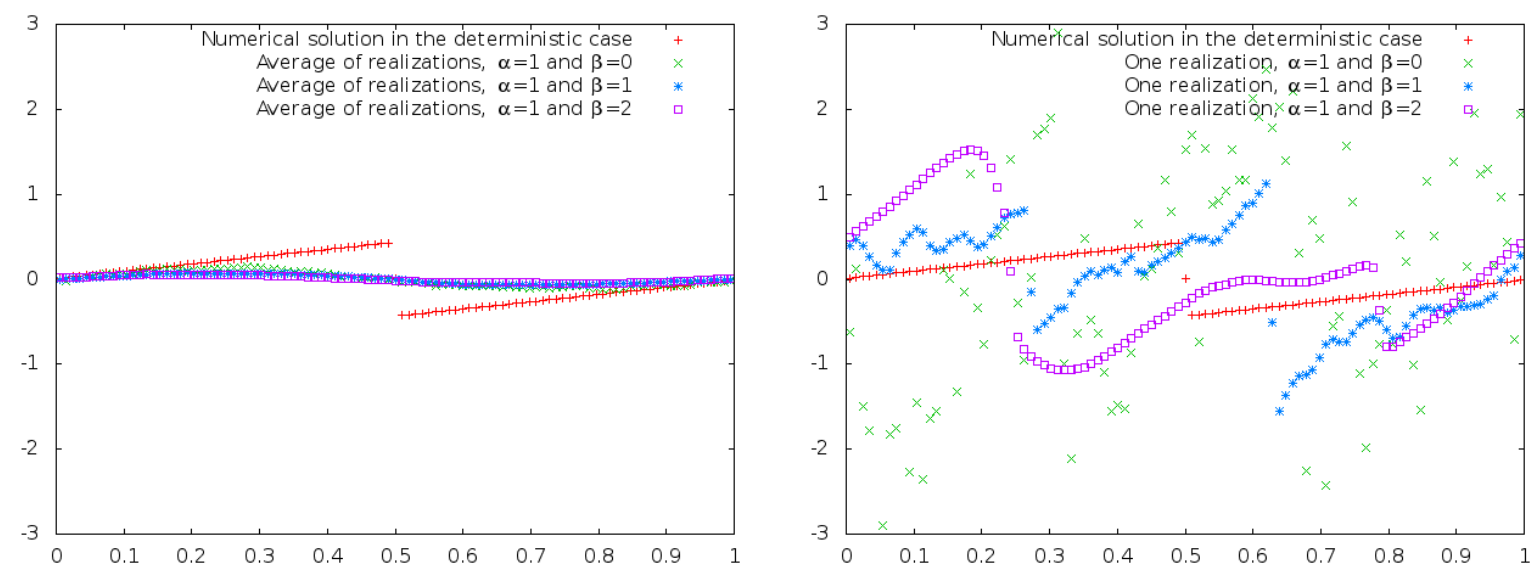

Figure 9. Empirical average (left) and one realization (right) at $t=1$ 

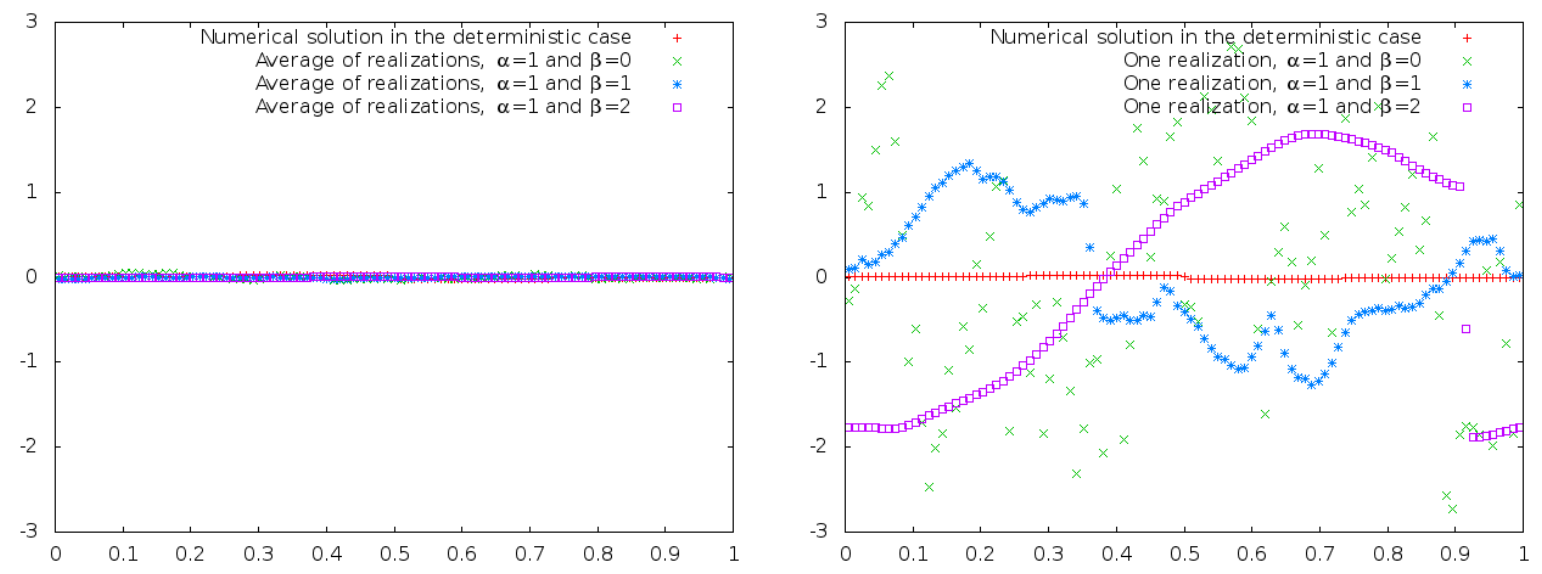

Figure 10. Empirical average (left) and one realization (right) at $t=20$

In Figure 11 we rescale again the comparisons at the times $t=1$ and $t=20$.

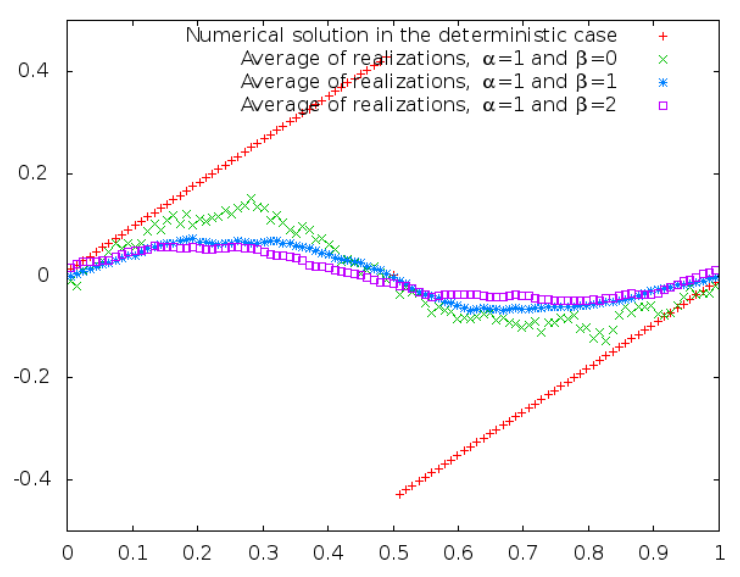

(A) $t=1$

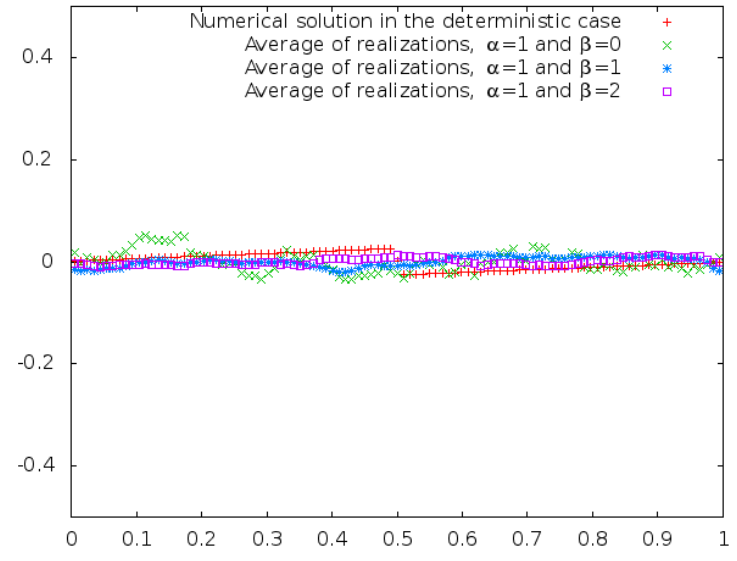

(B) $t=20$

FIGURE 11. Rescaled graphs for $t=1$ and $t=20$

Numerical solutions of the periodic inviscid Burgers equation (1) look quite different according to the space regularity of the stochastic source term.

Whereas single realizations look like discontinuous functions in the case that $\beta=1$, they do not even look like functions in the case that $\beta=0$.

We remark that the average of the realizations, also on a large time interval, is very close to the deterministic solution, namely the solution of the partial differential equation where the source term $g$ is such that $g=0$. However, this average appears to smoothen the discontinuities, so that the addition of a stochastic right-hand-side seems to have a similar effect as the addition of a small diffusion term.

We now compare the ways in which the expectation and the variance converge towards their stationary values when the parameters $\alpha$ and $\beta$ vary. In particular we observe in Figure 12 faster convergence in time as $\alpha$ and $\beta$ increase, and larger variance in the large time limit as $\alpha$ increases and $\beta$ decreases; of course, this is precisely as it could be expected. 

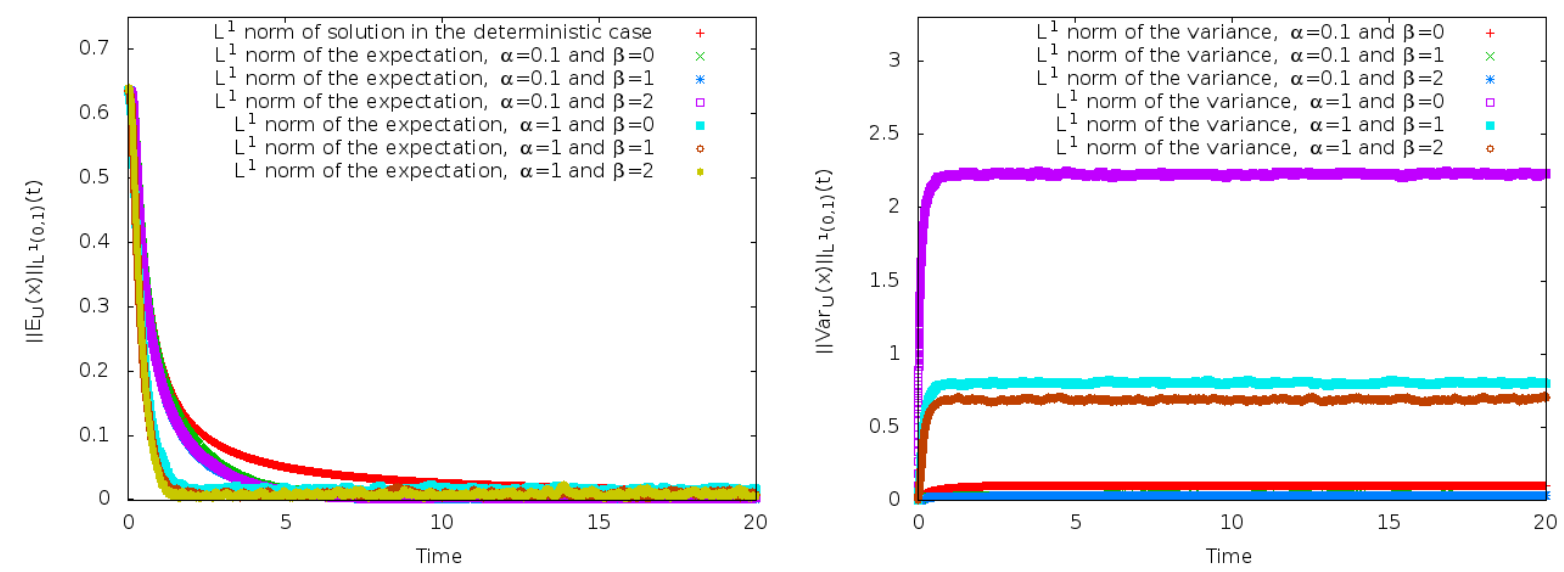

FIGURE 12. $L^{1}$-norm of the expectation (left) and of the variance (right) of the solution

\section{Some CONCLUSIONS}

We have considered cases of a white noise in space and time $(\alpha=0.1, \beta=0)$ and that of a smoother in space version $(\alpha=0.1, \beta=1$ and $\alpha=0.1, \beta=2)$. In those cases, the average of the realizations is a good approximation of the deterministic solution and as time tends to infinity it converges to the space-average of the initial function [7]. However, while the deterministic solution is discontinuous, the average of the realizations smoothens it out.

We have also considered corresponding cases with a larger amplitude, namely $(\alpha=1, \beta=0, \alpha=1, \beta=1$ and $\alpha=1, \beta=2$ ); then our numerical results for one realization are very dispersed. The average smoothens the deterministic solution and goes faster to equilibrium than in the cases where $\alpha=0.1$. However, when $\beta=0$, a single realization at $t=20$ does not seem to be a function anymore, which is consistent with the fact that the existence proof of an invariant measure for the solution of the continuous problem does not hold in this case.

Our results are still far from complete. A forthcoming work will also involve a numerical study of the limit $I \rightarrow \infty$, as well as a more detailed study of single realizations. We also propose to compute various distribution functions.

Acknowledgement. The authors would like to thank Henk Hilhorst, Hendrik Weber, Christine Keribin and Frédéric Lagoutière for helpful discussions. They also acknowledge EDF R\&D/Saint-Venant Laboratory which has supported Yueyuan Gao for taking part in CEMRACS 2013.

\section{APPENDIX: THE NUMERICAL APPROXIMATION OF THE NOISE}

We recall the numerical scheme:

$$
u_{i}^{n+1}=u_{i}^{n}-\frac{\Delta t}{\Delta x}\left(F_{i+\frac{1}{2}}^{n}-F_{i-\frac{1}{2}}^{n}\right)+\alpha \Delta t \sqrt{\frac{1}{\Delta t \Delta x}} G_{i}^{n} \quad \forall n \in \mathbb{N} \quad \forall i \in\{1, \ldots, I\},
$$

and propose to study the the formula

$$
G_{i}^{n}=\sqrt{\frac{2}{I}}\left(\sum_{k=1}^{\frac{I-1}{2}}\left(\frac{C_{k}^{n}}{k^{\beta}} \cos (2 \pi k \Delta x i)-\frac{S_{k}^{n}}{k^{\beta}} \sin (2 \pi k \Delta x i)\right)\right),
$$

where I is an odd number, and $C_{k}^{n}$ and $S_{k}^{n}$ follow the Gaussian law $\mathcal{N}(0,1)$ for fixed $k$ and $n$. In the following, we note $x_{i}:=\Delta x i$ and $x_{j}:=\Delta x j$.

We first check the conservation property, namely that $\sum_{i=1}^{I} G_{i}^{n}=0$ for all $n \in \mathbb{N}$. Indeed

$$
\begin{aligned}
\sum_{i=1}^{I} G_{i}^{n} & =\sum_{i=1}^{I} \sqrt{\frac{2}{I}}\left(\sum_{k=1}^{\frac{I-1}{2}}\left(\frac{C_{k}^{n}}{k^{\beta}} \cos \left(2 \pi k x_{i}\right)-\frac{S_{k}^{n}}{k^{\beta}} \sin \left(2 \pi k x_{i}\right)\right)\right) \\
& =\sqrt{\frac{2}{I}} \sum_{k=1}^{\frac{I-1}{2}} \frac{1}{k^{\beta}} C_{k}^{n} \sum_{i=1}^{I} \cos \left(2 \pi k x_{i}\right)-\sqrt{\frac{2}{I}} \sum_{k=1}^{\frac{I-1}{2}} \frac{1}{k^{\beta}} S_{k}^{n} \sum_{i=1}^{I} \sin \left(2 \pi k x_{i}\right) .
\end{aligned}
$$


In order to compute $\sum_{i=1}^{I} \cos \left(2 \pi k x_{i}\right)$ and $\sum_{i=1}^{I} \sin \left(2 \pi k x_{i}\right)$, we recall the Dirichlet kernel:

$$
D_{n}(2 \pi x)=\sum_{p=-n}^{n} e^{2 \pi \mathbf{i} p x}=1+2 \sum_{p=1}^{n} \cos (2 \pi p x)=\frac{\sin \left(\left(n+\frac{1}{2}\right) 2 \pi x\right)}{\sin \left(\frac{2 \pi x}{2}\right)}
$$

where $\mathbf{i}$ is the imaginary unit. We deduce from (20) the equality:

$$
\sum_{p=1}^{n} \cos (2 \pi p x)=\left(\frac{\sin \left(\left(n+\frac{1}{2}\right) 2 \pi x\right)}{\sin \left(\frac{2 \pi x}{2}\right)}-1\right) \cdot \frac{1}{2},
$$

so that

$$
\sum_{i=1}^{I} \cos \left(2 \pi k x_{i}\right)=\sum_{i=1}^{I} \cos \left(2 \pi i \frac{k}{I}\right)=\left(\frac{\sin \left(\left(I+\frac{1}{2}\right) \frac{2 \pi k}{I}\right)}{\sin \left(\frac{2 \pi k}{2 I}\right)}-1\right) \cdot \frac{1}{2} .
$$

Since $\sin \left(\left(I+\frac{1}{2}\right) \frac{2 \pi k}{I}\right)=\sin \left(\frac{2 \pi k}{2 I}+2 \pi k\right)=\sin \left(\frac{2 \pi k}{2 I}\right)$, we deduce from $(22)$ that $\sum_{i=1}^{I} \cos \left(2 \pi k x_{i}\right)=(1-1) \cdot \frac{1}{2}=0$.

Next we check that $\sum_{i=1}^{I} \sin \left(2 \pi k x_{i}\right)=0$

$$
\begin{aligned}
\sum_{i=1}^{I} \sin \left(2 \pi k x_{i}\right) & =\sum_{i=1}^{I} \sin \left(2 \pi k \frac{i}{I}\right) \\
& =\sum_{i=1}^{\frac{I-1}{2}}\left[\sin \left(2 \pi k \frac{i}{I}\right)+\sin \left(2 \pi k \frac{I-i}{I}\right)\right]+\sin \left(2 \pi k \frac{I}{I}\right) \\
& =\sum_{i=1}^{\frac{I-1}{2}}\left[\sin \left(2 \pi k \frac{i}{I}\right)+\sin \left(2 \pi k \frac{I}{I}-2 \pi k \frac{i}{I}\right)\right] \\
& =\sum_{i=1}^{\frac{I-1}{2}}\left[\sin \left(2 \pi k \frac{i}{I}\right)-\sin \left(2 \pi k \frac{i}{I}\right)\right] \\
& =0
\end{aligned}
$$

We conclude that $\sum_{i=1}^{I} G_{i}^{n}=0$ for all $n \in \mathbb{N}$ and $\beta \geq 0$.

Next, we compute $\mathrm{E}\left(G_{i}^{n} G_{j}^{m}\right)$ in the case of the white noise in space, namely when $\beta=0$. We have that:

$$
\begin{aligned}
G_{i}^{n} G_{j}^{m}= & \frac{2}{I}\left(\sum_{k=1}^{\frac{I-1}{2}}\left\{C_{k}^{n} \cos \left(2 \pi k x_{i}\right)-S_{k}^{n} \sin \left(2 \pi k x_{i}\right)\right\}\right)\left(\sum_{l=1}^{\frac{I-1}{2}}\left\{C_{l}^{m} \cos \left(2 \pi l x_{j}\right)-S_{l}^{m} \sin \left(2 \pi l x_{j}\right)\right\}\right) \\
= & \frac{2}{I} \sum_{k=1}^{\frac{I-1}{2}} \sum_{l=1}^{\frac{I-1}{2}}\left(C_{k}^{n} C_{l}^{m} \cos \left(2 \pi k x_{i}\right) \cos \left(2 \pi l x_{j}\right)+S_{k}^{n} S_{l}^{m} \sin \left(2 \pi k x_{i}\right) \sin \left(2 \pi l x_{j}\right)\right. \\
& \left.-C_{k}^{n} S_{l}^{m} \cos \left(2 \pi k x_{i}\right) \sin \left(2 \pi l x_{j}\right)-S_{k}^{n} C_{l}^{m} \sin \left(2 \pi k x_{i}\right) \cos \left(2 \pi l x_{j}\right)\right) .
\end{aligned}
$$

We recall that $S_{k}^{n}$ and $C_{l}^{m}$ are independent random variables for all $k$ and $l$, which implies that

$$
\mathrm{E}\left(S_{k}^{n} C_{l}^{m}\right)=\mathrm{E}\left(S_{k}^{n}\right) \mathrm{E}\left(C_{l}^{m}\right)
$$

moreover also remembering that both

$$
S_{k}^{n} \sim \mathcal{N}(0,1) \text { and } C_{l}^{m} \sim \mathcal{N}(0,1)
$$

we deduce from (25)

$$
\mathrm{E}\left(S_{k}^{n} C_{l}^{m}\right)=0
$$

and that

$$
\mathrm{E}\left(C_{k}^{n} S_{l}^{m}\right)=0
$$

as well. Similarly, if $k \neq l$ or $n \neq m, C_{k}^{n}$ and $C_{l}^{m}$ are i.i.d. random variables, so that

$$
\mathrm{E}\left(C_{k}^{n} C_{l}^{m}\right)=0 \quad \text { if } \quad k \neq l \text { or } n \neq m
$$

and

If $m=n$ and $k=l$, then in view of $(26)$

$$
\mathrm{E}\left(S_{k}^{n} S_{l}^{m}\right)=0 \quad \text { if } \quad k \neq l \text { or } n \neq m .
$$

$$
\mathrm{E}\left(C_{k}^{n} C_{l}^{m}\right)=\mathrm{E}\left(C_{k}^{n} \cdot C_{k}^{n}\right)=\operatorname{Var}\left(C_{k}^{n}\right)-\left(\mathrm{E}\left(C_{k}^{n}\right)\right)^{2}=1
$$


and we have a similar result for $\mathrm{E}\left(S_{k}^{n} S_{l}^{m}\right)$.

We conclude that $\mathrm{E}\left(C_{k}^{n} C_{l}^{m}\right)=\mathrm{E}\left(S_{k}^{n} S_{l}^{m}\right)=\delta_{m, n} \delta_{k, l}$. We deduce from (24) that:

$$
\begin{aligned}
\mathrm{E}\left(G_{i}^{n} G_{j}^{m}\right) & =\delta_{m, n} \frac{2}{I} \sum_{k=1}^{\frac{I-1}{2}} \sum_{l=1}^{\frac{I-1}{2}} \delta_{k, l}\left(\cos \left(2 \pi k x_{i}\right) \cos \left(2 \pi l x_{j}\right)+\sin \left(2 \pi k x_{i}\right) \sin \left(2 \pi l x_{j}\right)\right) \\
& =\delta_{m, n} \frac{2}{I} \sum_{k=1}^{\frac{I-1}{2}}\left(\cos \left(2 \pi k x_{i}\right) \cos \left(2 \pi k x_{j}\right)+\sin \left(2 \pi k x_{i}\right) \sin \left(2 \pi k x_{j}\right)\right) \\
& =\delta_{m, n} \frac{2}{I} \sum_{k=1}^{\frac{I-1}{2}} \cos \left(2 \pi k\left(x_{i}-x_{j}\right)\right) \\
& =\delta_{m, n} \frac{2}{I} \sum_{k=1}^{\frac{I-1}{2}} \frac{e^{2 \pi \mathbf{i} k\left(x_{i}-x_{j}\right)}+e^{-2 \pi \mathbf{i} k\left(x_{i}-x_{j}\right)}}{2} \\
& =\delta_{m, n} \frac{1}{I} \sum_{k=1}^{\frac{I-1}{2}}\left(e^{2 \pi \mathbf{i} k\left(x_{i}-x_{j}\right)}+e^{-2 \pi \mathbf{i} k\left(x_{i}-x_{j}\right)}\right) \\
& =\delta_{m, n} \frac{1}{I} \sum_{k=-\frac{I-1}{2}, k \neq 0}^{\frac{I-1}{2}} e^{2 \pi \mathbf{i} k\left(x_{i}-x_{j}\right)} \\
& =\frac{\delta_{m, n}}{I}\left[\sum_{k=-\frac{I-1}{2}}^{\frac{I-1}{2}} e^{2 \pi \mathbf{i} k\left(x_{i}-x_{j}\right)}-1\right]
\end{aligned}
$$

Next we consider the term $\sum_{k=-\frac{I-1}{2}}^{\frac{I-1}{2}} e^{2 \pi \mathbf{i} k\left(x_{i}-x_{j}\right)}$ :

if $i=j, x_{i}-x_{j}=0$, then

$$
\sum_{k=-\frac{I-1}{2}}^{\frac{I-1}{2}} e^{2 \pi \mathbf{i} k\left(x_{i}-x_{j}\right)}=I \cdot 1=I,
$$

but if $i \neq j, x_{i}-x_{j} \neq 0$, then we use again the Dirichlet kernel (20) to obtain:

$$
\begin{aligned}
\sum_{k=-\frac{I-1}{2}}^{\frac{I-1}{2}} e^{2 \pi \mathrm{i} k\left(x_{i}-x_{j}\right)} & =\frac{\sin \left(\left(\frac{I-1}{2}+\frac{1}{2}\right) \cdot 2 \pi\left(x_{i}-x_{j}\right)\right)}{\sin \left(\pi\left(x_{i}-x_{j}\right)\right)} \\
& =\frac{\sin (\pi I(i-j))}{\sin \left(\pi\left(x_{i}-x_{j}\right)\right)} \\
& =0 .
\end{aligned}
$$

Thus

Finally we deduce that

$$
\sum_{k=-\frac{I-1}{2}}^{\frac{I-1}{2}} e^{2 \pi \mathbf{i} k\left(x_{i}-x_{j}\right)}=I \delta_{i, j}
$$

$$
\begin{aligned}
\mathrm{E}\left(G_{i}^{n} G_{j}^{m}\right) & =\frac{\delta_{m, n}}{I}\left[\sum_{k=-\frac{I-1}{2}}^{\frac{I-1}{2}} e^{2 \pi \mathrm{i} k\left(x_{i}-x_{j}\right)}-1\right] \\
& =\frac{\delta_{m, n}}{I}\left[I \delta_{i, j}-1\right] \\
& =\delta_{m, n}\left[\delta_{i, j}-\frac{1}{I}\right]
\end{aligned}
$$

so that $G_{i}^{n}$ corresponds to a discrete white noise.

\section{REFERENCES}

[1] Aureli Alabert and István Gyöngy, On numerical approximation of stochastic Burgers' equation, From Stochastic Calculus to Mathematical Finance, Springer Berlin Heidelberg, 2006, pp. 1-15. 
[2] D. Amadori and D. Serre, Asymptotic behavior of solutions to conservation laws with periodic forcing, J. Hyperbolic Differ. Equ. 3 (2006), no. 2, 387-401.

[3] C. Bauzet, J. Charrier and T. Gallouët, Convergence of flux-splitting finite volume schemes for hyperbolic scalar conservation laws with a multiplicative stochastic perturbation, To be published.

[4] Alexandre Boritchev Equation de Burgers généralisée force aléatoire et à viscosité petite - Generalised Burgers equation with random force and small viscosity, Ecole Polytechnique, 8 October 2012.

[5] François Bouchut, Nonlinear stability of finite volume methods for hyperbolic conservation laws and well-balanced schemes for sources, Frontiers in Mathematics, Birkhäuser Verlag, Basel, 2004. MR MR2128209 (2005m:65002)

[6] C. Chainais-Hillairet Finite volume schemes for a nonlinear hyperbolic equation. Convergence towards the entropy solution and error estimate M2AN, Vol. 33, No 1, 1999, p.129-156.

[7] Arnaud Debussche and Julien Vovelle, Long-time behavior in scalar conservation laws, Diff. and Int. Eq. 22 (2009), no. 3-4, 225-238.

[8] Arnaud Debussche and Julien Vovelle. Invariant measure of scalar first-order conservation laws with stochastic forcing, Diff. and Int. Eq., 22(3-4):225-238, 2013.

[9] Arnaud Debussche and Julien Vovelle. Scalar conservation laws with stochastic forcing, REVISED VERSION, Journal of Functional Analysis, 259(4):1014-1042, 2010. 2013 REVISED VERSION http://hal.archives-ouvertes.fr/hal-00451641.

[10] Weinan E, K. Khanin, A. Mazel and Ya. Sinai, Invariant measures for Burgers equation with stochastic forcing, Annals of Mathematics 151 (2000), no. 3, 877-960 (English).

[11] Weinan E, Konstantin Khanin, Alexandre Mazel and Yakov Sinai, Probability distribution functions for the random forced Burgers equation, Phys. Rev. Lett. 78 (1997), 1904-1907.

[12] Weinan E and Eric Vanden Eijnden, Asymptotic theory for the probability density functions in Burgers turbulence, Phys. Rev. Lett. 83 (1999), 2572-2575.

[13] Robert Eymard, Thierry Gallouët and Raphaèle Herbin, Finite volume methods, Handbook of numerical analysis, Vol. VII, Handb. Numer. Anal., VII, North-Holland, Amsterdam, 2000, pp. 713-1020. MR MR1804748 (2002e:65138)

[14] Jin Feng and David Nualart, Stochastic scalar conservation laws, Journal of Functional Analysis 255 (2008), no. 2, $313-373$.

[15] Martin Hairer and Jochen Voss, Approximations to the stochastic Burgers equation, Journal of Nonlinear Science 21 (2011), no. 6, 897.

[16] Peter E. Kloeden and Eckhard Platen, Numerical Solution of Stochastic Differential Equations, Springer-Verlag Berlin Heidelberg 1992.

[17] S. N. Kružkov, First order quasilinear equations with several independent variables., Mat. Sb. (N.S.) 81 (123) (1970), $228-255$. MR 0267257 (42 \#2159)

[18] Randall J. LeVeque, Finite volume methods for hyperbolic problems, Cambridge Texts in Applied Mathematics, Cambridge University Press, Cambridge, 2002. MR MR1925043 (2003h:65001)

[19] S. P. Meyn and R. L. Tweedie, Markov chains and stochastic stability, Communications and Control Engineering Series, SpringerVerlag London Ltd., London, 1993, Available at http://probability.ca/MT/. MR 1287609 (95j:60103)

[20] G. N. Milstein and M. V. Tretyakov Stochastic numerics for mathematical physics Springer-Verlag, Berlin, 2004.

[21] S. Osher, Riemann solvers, the Entropy Condition, and Difference approximations, SIAM J. Num. Analysis 21 (1984), $217-235$. 